\title{
Study of two photon production process in proton-proton collisions at $216 \mathrm{MeV}$
}

\author{
A.S.Khrykin \\ Joint Institute for Nuclear Research, Dubna, 141980 Russia \\ Email: Khrykin@nusun.jinr.dubna.su
}

\begin{abstract}
The energy spectrum for high energy $\gamma$-rays $\left(E_{\gamma} \geq 10 \mathrm{MeV}\right)$ from the process $p p \rightarrow \gamma \gamma X$ emitted at $90^{\circ}$ in the laboratory frame has been measured at $216 \mathrm{MeV}$. The resulting photon energy spectrum extracted from $\gamma-\gamma$ coincidence events consists of a narrow peak $(5.3 \sigma)$ at a photon energy of about $24 \mathrm{MeV}$ and a relatively broad peak $(3.5 \sigma)$ in the energy range of $(50-70) \mathrm{MeV}$. This behavior of the photon energy spectrum is interpreted as a signature of the exotic dibaryon resonance $d_{1}^{\star}$ with a mass of about $1956 \mathrm{MeV}$ which is assumed to be formed in the radiative process $p p \rightarrow \gamma d_{1}^{\star}$ followed by its electromagnetic decay via the $d_{1}^{\star} \rightarrow p p \gamma$ mode. The experimental spectrum is compared with those obtained by means of Monte Carlo simulations.
\end{abstract}

\section{Introduction}

The process $p p \rightarrow p p \gamma \gamma$ at energy below the pion production threshold $(\pi N N)$ is still poorly explored both theoretically and experimentally. It was first suggested not long ago as a sensitive probe of the possible existence of $N N$-decoupled nonstrange dibaryon resonances [1], 2]. These are two-baryon states ${ }^{2} B$ with zero strangeness and exotic quantum numbers $I\left(J^{P}\right)[I$ is the isospin, $J$ is the total spin, and $P$ is the parity of a dibaryon state], for which the strong decay ${ }^{2} B \rightarrow p p$ is either strictly forbidden by the Pauli principle [for the states with $I\left(J^{P}\right)=1\left(1^{+}, 3^{+}\right.$, etc. $)$] or is strongly suppressed by the isospin selection rules (for the states with $I=2$ ). Such dibaryon states cannot be simply bound systems of two nucleons, and a proof of their existence would have consequences of fundamental significance for the theory of strong interactions [3, 4, 5].

If the $N N$-decoupled dibaryons exist in nature, then the $p p \gamma \gamma$ process may proceed, at least partly, through the mechanism that directly involves the radiative excitation $p p \rightarrow \gamma^{2} B$ and decay ${ }^{2} B \rightarrow \gamma p p$ modes of these states. In $p p$ collisions at energies below the $\pi N N$ threshold, these production and decay modes of the $N N$-decoupled dibaryon resonances with masses 
$M_{R} \leq 2 m_{p}+m_{\pi}$ would be unique or dominant. Since such dibaryons may decay mainly into the $p p \gamma$ state, their widths should be very narrow $(\leq 1 \mathrm{keV})$. The simplest and clear way of revealing them is to measure the photon energy spectrum of the reaction $p p \gamma \gamma$. The presence of an $N N$ decoupled dibaryon resonance would reveal itself in this energy spectrum as a narrow peak associated with the formation of the resonance and a relatively broad peak originating from its three-particle decay. In the center-of-mass system, the position of the narrow peak $\left(E_{R}\right)$ is determined by the energy of colliding nucleons $(W=\sqrt{s})$ and the mass of this dibaryon resonance as $E_{R}=\left(W^{2}-M_{R}^{2}\right) / 2 W$. An essential feature of the $p p \gamma \gamma$ process at an energy below the $\pi N N$ threshold is that, apart from the resonant mechanism in question, there should only be one more source of photon pairs. This is the double $p p$ bremsstrahlung reaction. But this reaction is expected to play a minor role. Indeed, it involves two electromagnetic vertices, so that one may expect that the $p p \gamma \gamma$-to- $p p \gamma$ cross section ratio should be of the order of the fine structure constant $\alpha$. However, the cross section for $p p \gamma$ is already small (the total cross section for the $p p \gamma$ reaction at energies of interest is a few $\mu b)$.

The preliminary experimental studies of the reaction $p p \rightarrow \gamma \gamma X$ at an energy of about $200 \mathrm{MeV}$ [6, (7] showed that the photon energy spectrum of this reaction had a peculiar structure ranging from about $20 \mathrm{MeV}$ to about 60 $\mathrm{MeV}$. This structure was interpreted as an indication of the possible existence of an $N N$-decoupled dibaryon resonance (later called $d_{1}^{\star}$ ) that is produced in the process $p p \rightarrow \gamma d_{1}^{\star}$ and subsequently decays via the $d_{1}^{\star} \rightarrow p p \gamma$ channel. Unfortunately, a relatively coarse energy resolution and low statistics did not allow us to distinguish the narrow $\gamma$ peak associated with the $d_{1}^{\star}$ production from the broad $\gamma$ peak due to its decay and, hence, to determine the resonance mass exactly. To clarify the situation with the dibaryon resonance $d_{1}^{\star}$, we have decided to measure the energy spectrum of the $p p \rightarrow p p \gamma \gamma$ reaction more carefully.

\section{The Experiment and Results}

The experiment was performed using the variable energy proton beam from the phasotron at the Joint Institute for Nuclear Research (JINR). The pulsed proton beam with an energy of about $216 \mathrm{MeV}$, an energy spread of about $1.5 \%$, and an intensity of about $3.6 \cdot 10^{8}$ protons $/ \mathrm{s}$ bombarded a liquid hydrogen target. Both $\gamma$ quanta of the reaction $p p \gamma \gamma$ were detected by two $\gamma$-ray detectors placed in a horizontal plane, symmetrically on either side of the

beam at a laboratory angle of $90^{\circ}$ with respect to the beam direction. The 
solid angles covered by the detectors were $43 \mathrm{msr}$ and $76 \mathrm{msr}$, respectively. To reject events induced by charged particles, plastic scintillators were put in front of each $\gamma$ detector. The electronics associated with the $\gamma$ detectors and the plastic scintillators together with the data acquisition system provided $\gamma-\gamma$ coincidence candidate events to be recorded on the hard disk of the computer. A further selection of events associated with the process $p p \rightarrow \gamma \gamma X$ was done during off-line data processing. The energy threshold for both the $\gamma$ detectors was set at about $7 \mathrm{MeV}$.

Measurements were done both for the target filled with liquid hydrogen and for the empty one. Data for the full and empty target were taken in two successive runs for $\sim 31 \mathrm{~h}$ and $\sim 21 \mathrm{~h}$, respectively. The integrated luminosity of about $8.5 \mathrm{pb}^{-1}$ was accumulated for the measurement with the full target. The photon energy spectrum of the process $p p \rightarrow \gamma \gamma X$ obtained after subtraction of the empty-target contribution from the spectrum measured with the full target is shown in Fig. 1. As can be seen, this spectrum consists of a narrow peak at a photon energy of about $24 \mathrm{MeV}$ and a relatively broad peak in the energy range from about 50 to about $70 \mathrm{MeV}$. The statistical significances for the narrow and the broad peaks are $5.3 \sigma$ and $3.5 \sigma$, respectively. The width (FWHM) of the narrow peak was found to be about $8 \mathrm{MeV}$. This width is comparable with that of the energy resolution of the experimental setup. The observed behavior of the photon energy spectrum agrees with a characteristic signature of the sought dibaryon resonance $d_{1}^{\star}$ that is formed and decays in the radiative process $p p \rightarrow \gamma d_{1}^{\star} \rightarrow p p \gamma \gamma$. In that case the narrow peak should be attributed to the formation of this dibaryon, while the broad peak should be assigned to its three-particle decay. Using the value for the energy of the narrow peak $E_{R} \sim 24 \mathrm{MeV}$, we obtained the $d_{1}^{\star}$ mass $M_{R} \sim 1956 \mathrm{MeV}$. The differential cross section for the resonance production of two photons emitted symmetrically at $\theta_{l a b}= \pm 90^{0}$ from the process $p p \rightarrow \gamma d_{1}^{\star} \rightarrow p p \gamma \gamma$ at an energy of $216 \mathrm{MeV}$ was estimated to be $\sim 9 \mathrm{nb} / \mathrm{sr}^{2}$.

Having assumed that the $p p \rightarrow \gamma d_{1}^{\star} \rightarrow p p \gamma \gamma$ process with the $d_{1}^{\star}$ mass of $1956 \mathrm{MeV}$ is the only mechanism of the reaction $p p \rightarrow p p \gamma \gamma$, we calculated the photon energy spectra of this reaction for a proton energy of $216 \mathrm{MeV}$. It was also assumed that the radiative decay of the $d_{1}^{\star}$ is a dipole $E 1(M 1)$ transition from the two-baryon resonance state to a $p p$ state in the continuum. The calculations were carried out with the help of Monte Carlo simulations which included the geometry and the energy resolution of the actual experimental setup. The photon energy spectra were calculated for two different scenarios of the $d_{1}^{\star}$ decay. The difference between them was that one of these scenarios took into account the final state interaction $(F S I)$ of two outgoing protons whereas in the other that interaction was switched off. Each of the scenarios imposed some restrictions on possible quantum numbers of the dibaryon state 


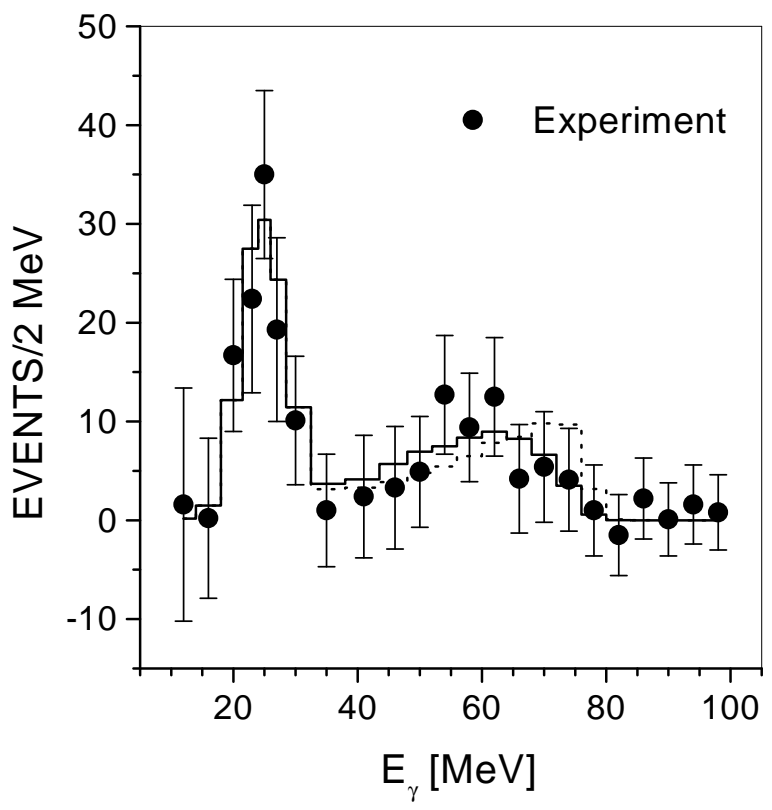

Figure 1: Experimentally observed energy spectrum for photons from the $p p \gamma \gamma$ process and energy spectra for photons from the process $p p \rightarrow \gamma d_{1}^{\star} \rightarrow \gamma \gamma p p$ calculated with the help of Monte Carlo simulations for two $d_{1}^{\star}$ decay scenarios: without the FSI (solid line) and with the FSI (dashed line).

in question. The scenario including the FSI implies that the final $p p$-system is in the singlet ${ }^{1} S_{0}$ state and consequently it should take place, in particular, for the isovector $1^{+}$dibaryon state (the simplest exotic quantum numbers), namely, $1^{+} \stackrel{M 1}{\longrightarrow} 0^{+}$. Moreover such a scenario can take place for any isotensor dibaryon state with the exception of the $0^{+}$or $0^{-}$state. At the same time, the scenario in which the FSI is switched off, is most likely to occur for the isotensor $0^{ \pm}$dibaryon state. The spectra calculated for these two decay scenarios and normalized to the total number of $\gamma-\gamma$ events observed in the present experiment are shown in Fig. 1. Comparison of these spectra with the experimental spectrum indicates that both the calculated spectra are in reasonable agreement with the experimental one within experimental uncertainties. In other words, the statistics of the experiment is insufficient to draw any firm conclusions in favor of one of these scenarios and thereby to limit possible quantum numbers of the observed dibaryon state.

Here it is important to note that the $d_{1}^{\star}$ with any possible set of quantum numbers $I\left(J^{P}\right)$ with the exception of $2\left(0^{ \pm}\right)$should mainly decay to the singlet ${ }^{1} S_{0}$ pp state. At the same time the outgoing protons from the process $p p \rightarrow$ 
$\gamma d_{1}^{\star} \rightarrow \gamma \gamma^{1} S_{0} p p$ would mainly be concentrated in a narrow angular cone near the direction of motion of incident protons [8]. We believe that this is why that this process was not found in the Uppsala $p p$ bremsstrahlung data [9].

\section{Conclusion}

The $\gamma$-ray energy spectrum for the $p p \rightarrow \gamma \gamma X$ reaction at a proton energy below the pion production threshold has been measured for the first time. The spectrum measured at an energy of about $216 \mathrm{MeV}$ for coincident photons emitted at an angle of $90^{\circ}$ in the laboratory frame clearly evidences the existence of the $N N$-decoupled dibaryon resonance $d_{1}^{\star}$ with a mass of $\sim 1956$ $\mathrm{MeV}$ that is formed and decays in the process $p p \rightarrow \gamma d_{1}^{\star} \rightarrow p p \gamma \gamma$. The data we have obtained, however, are still incomplete, and additional careful studies of the reaction $p p \rightarrow p p \gamma \gamma$ are needed to get proper parameters (mass, width, spin, etc.) of the observed dibaryon state.

\section{References}

[1] S.B. Gerasimov and A.S. Khrykin, Mod. Phys. Lett. A8, 2457(1993).

[2] S.B. Gerasimov, S.N. Ershov, A.S. Khrykin, Phys. At. Nucl. 58, 844(1995).

[3] P.J. Mülders, A.T. Aerts, and J.J. de Swart, Phys. Rev. D 21, 2653(1980).

[4] L.A. Kondratyuk, B.V. Martem'yanov, and M.G. Shchepkin, Sov. J. Nucl. Phys. 45, 776(1987).

[5] V.B. Kopeliovich, Phys. At. Nucl. 58, 1237(1995).

[6] A.S.Khrykin, inProceeding of the XIV International conference on particles and nuclei (PANIC96), Willianisburg, Virginia,USA,1996, ed. by Carl E.Carlson and John J.Domingo, (World Scientific, Singapore), p.533.

[7] A.S.Khrykin, in Proceeding of the Seventh International Symposium on Meson-Nucleon Physics and the Structure of the Nucleon, Vancouver, British Colombia, Canada, 1997, ed. by D.Drechsel, G.Höhler, W.Kluge, and B.M.K.Nefkens, (TRIUMF, Vancouver, 1997), p.250.

[8] A. S. Khrykin et al., Phys. Rev. C64,034002(2001). 
[9] H.Calén et al., Phys. Lett. B427, 248(1998). 